\title{
ANÁLISE DA PRESENÇA DE MICROPLÁSTICO EM BIVALVES (PERNA PERNA): UM ESTUDO DE CASO EM MATINHOS, LITORAL DO PARANÁ
}

\section{Juliana Araújo Machado}

Universidade Estadual do Paraná

Paranaguá - PR - Brasil

E-mail: contato.juliana01@gmail.com

Orcid: https://orcid.org/0000-0002-3836-2969

\section{Suzane de Oliveira}

Universidade Federal do Paraná, Setor Litoral

Matinhos - PR - Brasil

E-mail: suzioliveirabb@hotmail.com

Orcid: https://orcid.org/0000-0002-3616-3018

\section{Mariana Gallucci Nazário}

Universidade Federal do Paraná, Setor Litoral

Matinhos - PR - Brasil

E-mail: mari.nazario@yahoo.com.br

Orcid: https://orcid.org/0000-0001-7205-1681

\section{Heloísa Fernandes}

Instituto Federal do Paraná, Campus Paranaguá

Paranaguá, Paraná, Brasil

E-mail: heloisa.fernandes@ifpr.edu.br

Orcid: https://orcid.org/0000-0002-4981-3710

\author{
Allan Paul Krelling \\ Instituto Federal do Paraná, Campus Paranaguá \\ Paranaguá, Paraná, Brasil \\ E-mail: allan.krelling@ifpr.edu.br \\ Orcid: https://orcid.org/ 0000-0002-0050-2049
}

Recebido em 30/09/2020 aprovado em 31/03/2021

DOI: http://dx.doi.org/10.5380/guaju.v7i1.76916

\section{Resumo}

Microplásticos são partículas plásticas com tamanho inferior a $5 \mathrm{~mm}$, conhecidos como os detritos plásticos mais abundantes no oceano. Atualmente, microesferas, fragmentos ou microfibras de plástico podem ser encontrados nos mares de todo planeta Terra, oriundos de diferentes fontes e polímeros. A ingestão destas partículas pela fauna marinha tem sido amplamente documentada, resultando na contaminação da cadeia alimentar. Além disso, devido aos riscos associados ao seu consumo pelos seres humanos, este trabalho tem como objetivo analisar 
e quantificar a presença de microplásticos em bivalves selvagens (Perna perna) presentes e comercializados em Matinhos, litoral do Paraná. Para isso, foi realizada a adaptação da metodologia de Phuong et al. (2018) para identificação da presença e ausência do microplástico, fazendo uso dos reagentes químicos $\mathrm{KOH}$ (Hidróxido de Potássio) e KI (lodeto de Potássio). O processo consiste na amostragem das espécies e submissão destes aos processos de digestão com $\mathrm{KOH}$ (10\%). As amostras individuais passaram por processos sequenciais de sedimentação (KOH $10 \%$ e KI 50\%) e, após cada etapa, filtração das frações sobrenadantes em filtro de fibra de vidro GF/F3 (1,2 $\mu \mathrm{m})$. Os filtros foram analisados com auxílio de estereoscópio e as partículas de microplásticos recuperadas foram contadas, fotografadas e separadas para posterior análise qualitativa. A análise do material filtrado indicou a existência de fragmentos plásticos no formato de fios/fibras, fragmentos, filamentos e esferas, comprovando a existência do material. Os resultados desta pesquisa poderão servir de subsídios para a tomada de decisão e definição de áreas prioritárias para estudos posteriores.

Palavras-chave: Lixo Marinho. Microplástico. Bivalves. Litoral Paranaense.

\title{
Analysis of Microplastic Presence in Bivalves (Perna Perna): A Case Study in Matinhos, Paraná Coast
}

\begin{abstract}
Microplastic is particles of plastic materials smaller than $5 \mathrm{~mm}$, numerically known as the most abundant plastic debris in the ocean. Nowadays, plastic microspheres, fragments, or microfibres can be found in the seas of the whole planet Earth, coming from different sources and polymers. Ingestion of these particles by marine fauna has been widely documented, resulting in the food chain widespread contamination. Considering the risks associated with its consumption, this paper aims to analyze and quantify the presence of microplastics in wild commercial bivalves (Perna perna) present on the coast of Paraná. For this, the methodology of Phuong et al. (2018) so that the presence of microplastic could be identified or not, using the chemical reagents $\mathrm{KOH}$ (Potassium Hydroxide) and KI (Potassium lodide). The process consists of sampling the species and submitting them to digestion processes with $\mathrm{KOH}(10 \%)$. The individual samples underwent sequential sedimentation processes $(10 \% \mathrm{KOH}$ and $50 \% \mathrm{KI})$, and after each step, the supernatant fractions were filtered fiberglass filter GF/ $F 3(1,2 \mu \mathrm{m})$. The filters were stereoscopically analyzed, and the recovered microplastic particles were counted, photographed, and separated for further qualitative analysis. The analysis of the filtered material confirmed the existence of plastic fragments in the shape of fibers, fragments, filaments, and spheres, proving the existence of the material and bringing data for possible further studies.
\end{abstract}

Keywords: Marine litter. Microplastic. Digestion. Bivalve. Paraná Coast. 


\section{Introdução}

A poluição por lixo marinho é um problema que não afeta apenas a esfera ambiental, mas também os campos da economia, saúde pública e degradação paisagística natural (NEVES, 2013). Atualmente, este tipo de poluição é considerado uma das maiores ameaças aos organismos marinhos, principalmente por sua facilidade de dispersão, o que a caracteriza como um poluente global (ASCER, 2015).

Segundo Jambeck et al. (2015), todos os anos são aportadas milhões de toneladas de resíduos sólidos, principalmente à base de plástico, nos oceanos de todo o mundo, originários de uma variedade de fontes terrestres e marítimas. Dentre os detritos encontrados no mar, a maior e, provavelmente, a mais prejudicial fração do lixo marinho tem composição plástica. Fatores como o baixo custo de produção, resistência à corrosão, facilidade de moldagem e outros, fazem com que o plástico seja cada vez mais utilizado na manufatura de diversos produtos, dentre eles, os produtos descartáveis que, em sua maioria, são descartados após o primeiro uso (ASCER, 2015; PHUONG, et al., 2018).

Os impactos da contaminação por plástico podem ser vistos de forma gradual em toda a fauna marinha desde a zooplâncton, meio-fauna e invertebrados, até mamíferos topo de cadeia trófica (BOTTERELL et al, 2019, GUSMÃO et al, 2016; SANTANA et al, 2016; ZANTIS et al, 2021), a ingestão de microplástico pode ser direta ou indireta, via cadeia trófica (SANTANA et al, 2017). Essa ingestão pode causar problemas físicos como inanição por falsa saciez e bloqueio do trato gastrointestinal (BARROS et al, 2020). O descarte inadequado de resíduos sólidos, a má gestão e a falta de alternativas para a sua reciclagem são alguns dos fatores que influenciam o crescimento da problemática (ASCER, 2015). Portanto, sendo o plástico um material não biodegradável, na medida em que é decomposto, seu processo de fragmentação forma partículas cada vez menores, chamadas de microplásticos.

Além disso, uma vez em ambiente aquático, o microplástico (MP) pode se dispersar em diferentes compartimentos ambientais (superfície da água, coluna da água e sedimento bentônico) devido a sua variedade de formatos e densidades, além de se tornar passível de ingestão por diversos organismos aquáticos, inclusive os bivalves. Além disso, por meio de ligação indireta da cadeia trófica, também podem ser ingeridos pelos seres humanos (WANG et al., 2019).

As partículas de MP presentes no oceano podem causar distúrbios na existência e interação de diversas espécies, assim como do zooplâncton (BOTTERELL et al., 2019), 
microalgas (PRATA et al., 2019), mexilhões (SCOTT et al., 2019), ostras (PHUONG et al., 2018), peixes (KARBALAEI et al., 2019), tartarugas (CARON et al., 2018), aves marinhas (ZHU et al., 2019), estando presentes nas águas (KOELMANS et al., 2019) e sedimentos (WANG et al., 2019), afetando assim todo o ecossistema marinho.

Quanto aos objetos de estudo, os bivalves correspondem a um grupo de animais filtradores conhecidamente afetados pela presença e exposição do MP, sendo estudados amplamente em todo o mundo (LI et al., 2015). Alguns moluscos desse gênero chegam a filtrar até 100 litros de água por dia, concentrando, em seus tecidos, grande quantidade de substâncias químicas, micro-organismos e resíduos orgânicos e inorgânicos (DAME, 1996 apud EVANGELISTA-BARRETO et al., 2008).

Em consonância a sua abundância, o consumo de ostras, mariscos e mexilhões é uma prática comum em regiões litorâneas do Brasil, sendo geralmente consumido cru no caso de ostras, e cozidos como os mexilhões e berbigões (SUPLICY, 2018). Por serem espécies que se alimentam por filtração da água, e por consumirem matéria orgânica e microalgas, os bivalves podem reter e acumular contaminantes, poluentes como mercúrio e outros metais pesados (MEYER et al., 1998; VIEIRA et al., 2021), bactérias patogênicas (E. coli) (MARTINEZ; OLIVEIRA, 2010; KOLM et al, 2008); e toxinas produzidas por microalgas como as Ostreopsis (TIBIRIÇÁ et al., 2019) e Dinophysis spp (ALVES et al., 2018) presentes na água e perigosas para o ser humano (SOUZA; PETCOV, 2013; BRASIL, 2014).

Nesse contexto, este trabalho tem como objetivo identificar a ingestão de MP por bivalves selvagens (Perna perna) em amostras comercializadas no Mercado Municipal de Pescados (município de Matinhos - litoral do Paraná).

\section{Fundamentação teórica}

\subsection{Lixo marinho}

Lixo marinho é qualquer material sólido processado ou manufaturado, que é descartado ou de alguma forma abandonado em ambiente marinho e costeiro (UNEP, 2019).

Segundo Galgani et al. (2010) citado por Ascer (2015): Faz parte também qualquer detrito (em especial elementos como madeira, plásticos, metais, vidros, borracha, roupas, papéis entre outros) que chega a esses ambientes, 
intencionalmente ou acidentalmente, por transporte natural como rios, ventos ou tempestade (p. 3).

A origem deste lixo marinho provém, essencialmente, das más práticas na gestão de resíduos, carência de infraestruturas urbanas como saneamento básico e do mau comportamento quanto às questões relacionadas aos resíduos (descarte incorreto, consumo inconsciente) das populações, sendo este um problema significativo para os países em processo de desenvolvimento (NEVES, 2013). Neves (2013) ainda ressalta que, segundo Derraik (2002), o plástico é o tipo de lixo marinho predominante no mundo, que segundo Galgani et al. (2000) cobre uma percentagem superior a 70\% nas plataformas e taludes continentais da Europa.

A reunião destes fatos nos alerta para o uso excessivo dos diversos tipos de materiais plásticos disponíveis em nosso mercado, principalmente os plásticos descartáveis, que são frequentemente utilizados nos grandes centros urbanos, como em bares e restaurantes, e, também, nas praias.

\subsection{Plástico}

Os plásticos são polímeros orgânicos que podem ser produzidos a partir de combustíveis fósseis, óleo, gás ou carvão (WANG et al., 2019). São chamados de plástico por exibirem a propriedade de plasticidade em sua forma (PHUONG et al., 2018).

Globalmente, milhões de toneladas de plástico são produzidos todos os anos e estima-se que, a cada ano, 10 a 20 milhões de toneladas entram nos oceanos, causando, anualmente, o equivalente a 13 bilhões de dólares em danos ao ecossistema marinho (UNEP, 2014). Essa produção desenfreada se deve a sua ampla diversidade de uso, grande durabilidade e leveza, o que o tornou um dos materiais mais procurados para a manufatura de diversos produtos, que vão desde tubulações até produtos hospitalares, abrangendo também móveis e brinquedos (ASCER, 2015).

As propriedades que tornam o plástico tão útil e presente no cotidiano humano, além das já descritas, incluem a sua estabilidade e resistência à degradação, características estas, que também são as principais causas que o tornam perigoso para o meio ambiente (FRIAS, 2010).

Segundo Andrady (2011), o plástico não é um material biodegradável e sua fragmentação acontece formando partículas cada vez menores, o que faz com que o tipo de 
poluição dispersa por ele só aumente com o tempo, resultando na produção de MP. Desta forma, a quantidade de MP é crescente devido à degradação de grandes itens de plástico, que acabam por se quebrar e se transformar em milhões de pedaços de MP.

\subsubsection{Microplástico}

Os microplásticos são definidos como pequenas partículas de materiais plásticos com tamanho inferior a $5 \mathrm{~mm}$, constituindo, numericamente, os detritos de plástico mais abundantes no oceano (GESAMP, 2016). No entanto, em pesquisa recente, Hartmann et al. (2019) sugere que essa definição deve ser revista e atualizada para partículas contendo de $1 \mathrm{a}<1000 \mu \mathrm{m}$ e que partículas contendo 1-10 mm passem a ser classificadas como mesoplástico e, a partir de $1 \mathrm{~cm}$, de macroplástico.

Encontrados em ambiente marinho, tipicamente em forma de esferas, fragmentos ou fios/fibras, os MPs podem ser compostos por diferentes polímeros, alguns mais densos que a água que tendem a afundar até o fundo do mar. Esses polímeros incluem poliamida (PA), poliéster (PET), policloreto de vinila (PVC) e acrílico (PMMA) entre outros. Outros são menos densos que a água do mar e são frequentemente encontradas flutuando na superfície, incluindo polietileno (PEAD e PEBD), polipropileno (PP), e poliestireno (PS) (SMITH et al., 2018).

As partículas de MP podem ser classificadas, segundo Boucher e Friot (2017), de acordo com sua origem, em primários e secundários. Como microplásticos primários citamse aqueles liberados diretamente no meio ambiente na forma de pequenas partículas, podendo ser originados da abrasão de tecidos têxteis sintéticos ou ainda, da abrasão de grandes objetos de plástico durante a fabricação, uso ou manutenção. Ainda, podem ser compostas por microesferas utilizadas em cosméticos, na forma de pellets, pequenos grânulos produzidos nas indústrias petroquímicas. Os microplásticos secundários são originários da degradação de plásticos maiores em pequenos fragmentos, quando expostos ao ambiente marinho. Dentre as os principais fatores de degradação e produção dos microplásticos secundários citam-se a fotodegradação, processo de intemperismos de resíduos não gerenciados (exemplo: sacolas plásticas, embalagens e materiais de pesca).

Os pellets possuem forma esférica (em tamanhos que variam de 1 a $5 \mathrm{~mm}$ ) ou forma de pó (que possuem tamanhos micrométricos), e são usados como matéria-prima da indústria plástica para a produção de inúmeros produtos ( ANDRADY, 2017). 
A presença de fragmentos de plásticos é uma realidade estabelecida nos oceanos, estuários e corpos d'água, surgindo como uma nova preocupação e objeto de estudo pelo seu contato e introdução na cadeia alimentar da biota marinha.

\subsection{Impactos do plástico no ecossistema marinho}

Os plásticos constituem diferentes tipos de polímeros, os quais são produzidos principalmente a partir de combustíveis fósseis como o petróleo, gás natural, ou carvão (WANG et al., 2019). Entretanto, estes raramente são utilizados na sua forma pura, podendo ser adicionados aos polímeros diferentes compostos químicos a fim de alterar suas características de origem e lhes conferir as qualidades necessárias para o seu uso final (DEANIN, 1975, apud ASCER, 2015). Dentre estes aditivos são encontrados corantes, estabilizadores, retardantes de chamas, plastificantes, entre outros. Segundo Boyer (1951) apud Ascer (2015), apesar da sua importância na composição do plástico estes aditivos podem ser liberados no ambiente, afetando os organismos que entram em contato. Portanto, o aumento desses polímeros aditivados nos oceanos pode levar a alterações fisiológicas dos organismos ali presentes.

Os detritos plásticos mostram-se, atualmente, como um problema de poluição persistente, com caráter difuso, afetando todos os oceanos do planeta, causando lesões e, em casos extremos, a morte de um amplo número de animais aquáticos e aves marinhas (FRIAS, 2010. Ao contrário dos grandes detritos plásticos, os microplásticos nos oceanos não podem ser facilmente encontrados, coletados para reciclagem ou descartados de forma ambientalmente adequada, não sendo incomum em regiões costeiras encontrar uma contagem de $10^{3}$ a $10^{4}$ microplásticos flutuantes por metro cúbico (ANDRADY, 2017).

Independentemente de sua forma (macro, meso ou micro) os plásticos afetam diretamente a vivência e as interações das cadeias alimentares dos animais marinhos, gerando também grandes prejuízos para as atividades marítimas, tais como a pesca, a navegação e a aquicultura. Na forma de macroplásticos podem impedir atividades das indústrias e danificar equipamentos pesqueiros (ASCER, 2015). Além disso, podem gerar perdas econômicas ao turismo (KRELLING et al., 2017). Já nas interações com os animais marinhos, podem causar emaranhamento, estrangulamento, ingestão e modificação dos habitats naturais, contato este que pode levá-los à morte (COSTA et al., 2015; BROWNE et al., 2015; GUEBERT-BARTHOLO, 2011). 
$\mathrm{Na}$ forma de microplásticos, afetam os mais diversos organismos, desde os produtores primários até o topo da cadeia alimentar, podendo ser inalados, absorvidos através da pele e ingeridos. Além do dano físico, seus contaminantes podem causar danos à fisiologia (molecular e celular) desses organismos (ASCER, 2015).

Estudos sobre ingestão e interação com MP realizados com espécies marinhas podem auxiliar na compreensão da qualidade e da saúde do ecossistema, uma vez que muitas espécies são consideradas como indicadoras de qualidade ambiental, como os mamíferos (ZANTIS et al., 2021) e bivalves (BRÅTE et al., 2018). Os primeiros representam a exposição direta e indireta de consumo de MP, enquanto os bivalves, devido à sua ampla distribuição mundial e por estarem presentes em nichos ecológicos vitais, são suscetíveis a absorção de MP, sendo consumidos tanto por predadores marinhos como por seres humanos (LI et al., 2019).

Ao analisar os estudos no litoral do Paraná, observa-se pouca literatura sobre microlixo, especialmente relacionando-o à biota. Os principais estudos realizados e publicados nesta região envolvem macrolixo (WIDMER e REIS, 2010; GUEBERT-BARTHOLO et al. 2011; POSSATTO et al. 2015; KRELLING et al. 2017a;2017b;2019) e aqueles que envolvem microlixo dedicaram-se especialmente a analisar a presença de pellets em praias (GORMAN et al., 2019), conforme apresentado no Quadro 1. Desta forma, a presença de MP em bivalves no litoral do Paraná é uma lacuna a ser explorada, pois permite não apenas identificar o microlixo no mar e águas circundantes (QU et al., 2018), mas também avaliar a qualidade dos frutos do mar de consumo humano na região. 
Quadro 1: Estudos sobre detritos plásticos no litoral do Paraná

\begin{tabular}{|c|c|c|c|}
\hline Referência & Local & Tipo de estudo & Itens encontrados \\
\hline WIDMER \& REIS, 2010 & Praias do Paraná & $\begin{array}{l}\text { Avaliação da eficácia da } \\
\text { distribuição e uso de } \\
\text { cinzeiros promocionais } \\
\text { nas praias }\end{array}$ & $\begin{array}{l}\text { Baixo descarte de cinzei- } \\
\text { ro nas praias, } 3,44 \text { pon- } \\
\text { tas de cigarro e outros } \\
\text { detritos plásticos por } \\
\text { cinzeiro promocional } \\
\end{array}$ \\
\hline $\begin{array}{l}\text { GUEBERT-BARTHOLO et } \\
\text { al. } 2011\end{array}$ & $\begin{array}{l}\text { Complexo Estuarino } \\
\text { de Paranaguá e praias } \\
\text { adjacentes }\end{array}$ & $\begin{array}{l}\text { Conteúdo estomacal de } \\
\text { tartaruga verde Chelonia } \\
\text { mydas }\end{array}$ & $\begin{array}{l}\text { Sacolas plásticas, } \\
\text { plástico rígido, nylon e } \\
\text { poliestireno }\end{array}$ \\
\hline POSSATTO et al. 2015 & $\begin{array}{l}\text { Complexo Estuarino de } \\
\text { Paranaguá }\end{array}$ & $\begin{array}{l}\text { Detritos sólidos coleta- } \\
\text { dos por rede de arrasto }\end{array}$ & $\begin{array}{l}291 \text { itens, sendo que } \\
92 \% \text { eram plásticos }\end{array}$ \\
\hline KRELLING et al. 2017a & $\begin{array}{l}\text { Complexo Estuarino de } \\
\text { Paranaguá }\end{array}$ & $\begin{array}{l}\text { Análise da distribuição } \\
\text { dos detritos sólidos an- } \\
\text { tropogênicos, ao longo } \\
\text { do gradiente estuarino }\end{array}$ & $\begin{array}{l}\text { O Complexo Estuarino } \\
\text { de Paranaguá tem perfil } \\
\text { de exportar os resíduos } \\
\text { sólidos flutuantes para } \\
\text { praias e áreas adjacentes } \\
\text { à sua desembocadura. } \\
\text { Dos } 924 \text { itens coletados, } \\
88,63 \% \text { são plásticos } \\
\text { (751) e poliestireno (77). }\end{array}$ \\
\hline KRELLING et al. 2017b & $\begin{array}{l}\text { Complexo Estuarino } \\
\text { de Paranaguá e praias } \\
\text { adjacentes }\end{array}$ & $\begin{array}{l}\text { Cálculo de perdas eco- } \\
\text { nômicas potenciais pela } \\
\text { presença de detritos } \\
\text { sólidos antropogênicos } \\
\text { em praias turísticas }\end{array}$ & $\begin{array}{l}\text { As perdas econômicas } \\
\text { pela presença de resídu- } \\
\text { os podem chegar a mais } \\
\text { de } 8 \text { milhões de dólares } \\
\text { para Pontal do Paraná/ } \\
\text { PR. }\end{array}$ \\
\hline KRELLING et al. 2019 & $\begin{array}{l}\text { Complexo Estuarino } \\
\text { de Paranaguá e praias } \\
\text { adjacentes }\end{array}$ & $\begin{array}{l}\text { Influência de fatores } \\
\text { ambientais sobre a } \\
\text { distribuição de detritos } \\
\text { sólidos }\end{array}$ & $\begin{array}{l}\text { O aumento da vazão é } \\
\text { um dos principais veto- } \\
\text { res de aumento no acú- } \\
\text { mulo de itens antropo- } \\
\text { gênicos em praias. Dos } \\
12.048 \text { itens coletados, } \\
\text { 83,5\% eram plásticos ou } \\
\text { espumas plásticas. }\end{array}$ \\
\hline GORMAN et al., 2019 & $\begin{array}{l}\text { Praias de Matinhos e } \\
\text { Pontal do Paraná }\end{array}$ & $\begin{array}{l}\text { Pellets coletados em } \\
\text { praias }\end{array}$ & $\begin{array}{l}\text { Presença de Conta- } \\
\text { minantes Orgânicos } \\
\text { Persistentes em pellets } \\
\text { ao longo das praias de } \\
\text { Matinhos e Pontal do } \\
\text { Paraná, independen- } \\
\text { temente do gradiente } \\
\text { espacial }\end{array}$ \\
\hline
\end{tabular}

Fonte: Elaborado pelos autores. 


\section{Metodologia}

\subsection{Aquisição das amostras e armazenamento}

Os mexilhões da espécie Perna perna foram comprados (2 L) no Mercado Municipal de Pescados do Município Matinhos (Figura 1), coletados em ambiente natural, no mês de junho de 2019. Após a compra, estes foram imediatamente transportados ao laboratório Acadêmico do Setor Litoral da UFPR, revestidas com papel alumínio, armazenadas em potes de vidro e congelados $\mathrm{a}-20^{\circ} \mathrm{C}$.

Figura 1: Localização do município de Matinhos - PR (à direita).
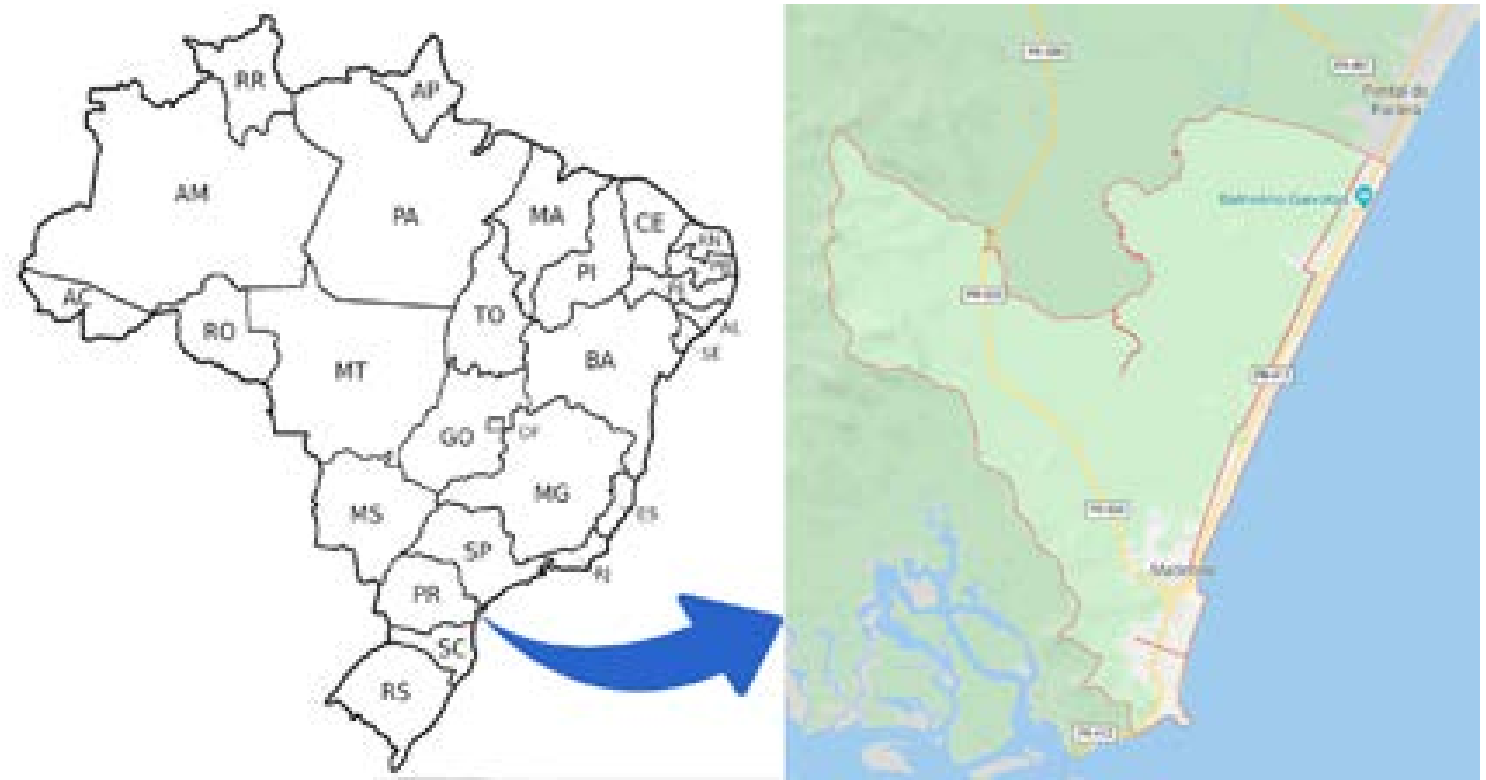

Fonte: Autor adaptado de INFOESCOLA (2021) e GOOGLE MAPS (2021)

\subsection{Divisão das amostras em agrupamentos}

Os mexilhões foram descongelados por aproximadamente uma hora e meia e, aleatoriamente, escolhidos e separados em uma bandeja revestida com papel kraft. Cada um dos mexilhões teve sua concha aberta com o auxílio de uma faca e o bisso removido com uma lâmina cirúrgica previamente limpa com água ultra pura. O processo de remoção do material da concha foi realizado com o auxílio de uma faca e lâmina cirúrgica (lavadas 
com água a cada troca de amostra) dentro de uma capela para evitar possível contato e contaminação das amostras com o MP presente no ar.

Os mexilhões foram medidos em centímetros para estratificar a amostra, e após analisar o sexo, foram pesados em balança de precisão (FA2104N) e divididos em pools de três indivíduos. Estes foram posteriormente colocados em Erlenmeyer e vedados com papel alumínio. No total foram realizadas cinco amostras e 1 branco, em duas análises (duplicata).

Para evitar a contaminação das amostras, todas as vidrarias utilizadas no processo foram submetidas a um banho ácido com HNO3 (Ácido Nítrico) e lavadas três vezes com água ultrapura antes de sua utilização. Durante todos os processos foram utilizadas luvas nitrílicas e jaleco 100\% algodão com o intuito de evitar a contaminação. A água ultrapura foi utilizada em todos os processos. Sempre que possível, as amostras eram cobertas com papel alumínio para evitar contaminação, assim como o uso de capela para a abertura e manuseio dos mexilhões (SANTANA et al., 2016).

\subsection{Digestão e sedimentação}

Após agrupadas as amostras, o processo de digestão foi realizado baseado na metodologia de Phuong et al. (2018) com adaptações. Foi adicionado no Erlenmeyer 50 $\mathrm{mL}$ de Hidróxido de Potássio - KOH (VETEC) a 10\% v/v a cada um dos frascos. Estes foram homogeneizados e submetidos à agitação magnética a $85 \mathrm{rpm}$, com aquecimento a $60^{\circ} \mathrm{C}$, durante 24 horas.

As amostras passaram por processos sequenciais de sedimentação em funis de decantação (Figura 2) para a separação das frações mais e menos densas, onde, após quatro horas, o material sobrenadante (menos denso) foi separado e reservado em novo Erlenmeyer de acordo com seus agrupamentos anteriores. No sedimento restante, foi acrescido $20 \mathrm{~mL}$ de lodeto de Potássio - KI (ÊXODO CIENTÍFICA) a 50\%, agitado manualmente e novamente colocado para sedimentação. Após quatro horas, o material sobrenadante foi retirado e colocado junto ao material reservado anteriormente. Para garantir que todo o MP foi retirado da vidraria, após o uso, todo o material foi lavado com água ultrapura e realizada filtração a vácuo. Tanto o $\mathrm{KOH}$ como o KI foram filtrados (GF/F3 de 1,2 $\mu \mathrm{m}$ ) a vácuo antes de serem utilizados. 
Figura 2 - Processo de sedimentação sequencial.

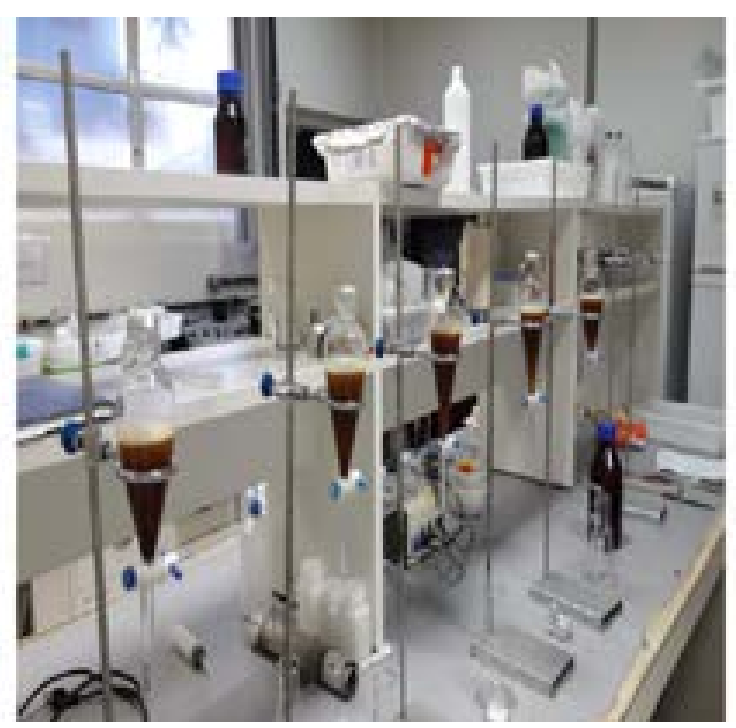

Fonte: Autor.

3.4 Filtração e análise dos microplásticos

Após os procedimentos descritos no item 3.3, realizou-se a filtração individual das amostras em filtros de vidro GF/F3 de 1,2 $\mu \mathrm{m}$ com o auxílio de uma bomba a vácuo. Os filtros obtidos foram separados individualmente em placas de Petri e analisados através de estereoscópio (LEICA) para verificar a quantidade de microplásticos filmes, fios/fibras, fragmentos e microesferas presentes. Todos os microplásticos foram caracterizados e contados de acordo com a cor, o tamanho e a forma (Figura 3).

Figura 3 - Filtro de vidro contendo microplásticos aderidos.

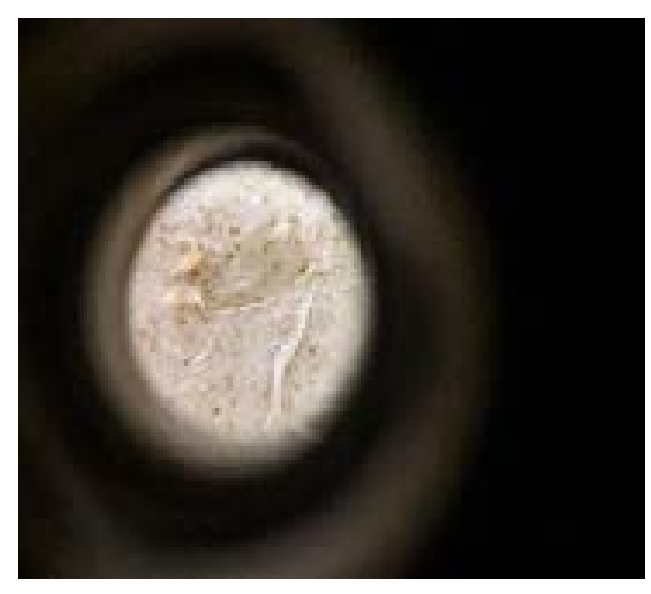

Fonte: Acervo pessoal. 
Esta metodologia foi utilizada da mesma forma em ambas as análises.

Quanto às adaptações metodológicas realizadas, pode-se destacar a mudança do tipo e tamanho (de $20 \mu \mathrm{m}$ para 1,2 $\mu \mathrm{m}$ ) do filtro utilizado, uso de pipeta para retirar o conteúdo digerido, lavagem do material após uso com água ultra pura e para a filtração à vácuo.

\section{Resultados e discussão}

\subsection{Dados descritivos das análises}

Conforme os dados apresentados nas Tabelas 1 e 2, foram encontrados em ambas as análises, majoritariamente, mexilhões do sexo feminino (24 espécimes), havendo uma incidência menor de mexilhões do sexo masculino (9 espécimes). Em relação ao tamanho, em fêmeas e machos, a variação foi pequena, podendo-se observar médias semelhantes em todas as amostras. Entretanto, seu peso apresentou uma variação maior, tendo no experimento 1 a média de 6,57 g e no experimento 2 a média de 5,72 g.

Tabela 1: Resultados da análise 1.

\begin{tabular}{|l|l|l|l|l|l|}
\hline Amostra & Sexo & $\begin{array}{l}\mathrm{N} \text { da } \\
\text { amostra }\end{array}$ & $\begin{array}{l}\text { Tamanho } \\
\text { médio }\end{array}$ & Peso médio $(\mathrm{g})$ & $\begin{array}{l}\text { Peso do Pool } \\
(\mathrm{g})\end{array}$ \\
\hline 1 & Feminino & $\mathrm{n}=3$ & $5,9 \mathrm{~cm} \pm 0,36$ & & \\
\hline 2 & Masculino & $\mathrm{n}=3$ & $6,5 \mathrm{~cm} \pm 0,35$ & $8,29 \pm 4,50$ & 24,8779 \\
\hline 3 & Feminino & $\mathrm{n}=3$ & $6,4 \mathrm{~cm} \pm 0,20$ & $8,59 \pm 2,07$ & 25,7822 \\
\hline 4 & Feminino & $\mathrm{n}=3$ & $5,6 \mathrm{~cm} \pm 0,11$ & $4,18 \pm 1,02$ & 12,5599 \\
\hline 5 & Feminino & $\mathrm{n}=3$ & $6,2 \mathrm{~cm} \pm 0,64$ & $5,25 \pm 0,94$ & 15,7422 \\
\hline 6 & Branco & & & & \\
\hline
\end{tabular}

Fonte: Elaborado pelos autores.

Nota: A Amostra 1 teve sua avaliação de peso comprometida, por isso foi excluído da análise 
Tabela 2 - Resultados da análise 2.

\begin{tabular}{|l|l|l|l|l|l|}
\hline Amostra & Sexo & $\begin{array}{l}\mathrm{N} \text { da } \\
\text { amostra }\end{array}$ & $\begin{array}{l}\text { Tamanho médio } \\
(\mathrm{cm})\end{array}$ & $\begin{array}{l}\text { Peso médio } \\
(\mathrm{g})\end{array}$ & $\begin{array}{l}\text { Peso do Pool } \\
(\mathrm{g})\end{array}$ \\
\hline 1 & Masculino & $\mathrm{n}=3$ & $5,07 \pm 0,98$ & $4,25 \pm 2,25$ & 12,7569 \\
\hline 2 & Feminino & $\mathrm{n}=3$ & $5,57 \pm 0,55$ & $4,61 \pm 0,54$ & 13,8327 \\
\hline 3 & Feminino & $\mathrm{n}=3$ & $5,67 \pm 0,76$ & $5,51 \pm 0,73$ & 16,5413 \\
\hline 4 & Feminino & $\mathrm{n}=3$ & $6,60 \pm 0,91$ & $5,64 \pm 1,76$ & 16,9300 \\
\hline 5 & Masculino & $\mathrm{n}=3$ & $5,83 \pm 1,30$ & $5,35 \pm 2,43$ & 16,0549 \\
\hline 6 & Branco & & & & \\
\hline
\end{tabular}

Fonte: Elaborado pelos autores.

\subsection{Microplástico encontrado nos mexilhões}

Ao total foram encontradas 83 partículas de MP, somando os resultados das duas análises. As formas encontradas foram fio/fibra (36 itens), filme (25 itens), fragmento (18 itens) e microesferas (4 itens). As Tabelas 3 e 4 demonstram individualmente, a frequência relativa e a frequência absoluta de MP de acordo com o tipo e cor predominante.

Tabela 3: Descrição de MP em mexilhões análise 1.

\begin{tabular}{|c|c|c|c|}
\hline & Quantidade & Cor predominante & $\begin{array}{l}\text { Frequência } \\
\text { relativa }\end{array}$ \\
\hline \multicolumn{4}{|l|}{ Amostra 1} \\
\hline $\begin{array}{l}\text { Fio/fibra } \\
\text { Filme plástico }\end{array}$ & $\begin{array}{l}n=6 \\
n=2\end{array}$ & $\begin{array}{l}\text { Azul, preto } \\
\text { Transparente }\end{array}$ & $\begin{array}{l}65 \% \\
25 \%\end{array}$ \\
\hline \multicolumn{4}{|l|}{ Amostra 2} \\
\hline $\begin{array}{l}\text { Fio/fibra } \\
\text { Filme plástico } \\
\text { Fragmento plástico }\end{array}$ & $\begin{array}{l}n=5 \\
n=3 \\
n=2\end{array}$ & $\begin{array}{l}\text { Preto } \\
\text { Transparente } \\
\text { Preto }\end{array}$ & $\begin{array}{l}50 \% \\
30 \% \\
20 \%\end{array}$ \\
\hline \multicolumn{4}{|l|}{ Amostra 3} \\
\hline $\begin{array}{l}\text { Fio/fibra } \\
\text { Filme plástico } \\
\text { Fragmento plástico }\end{array}$ & $\begin{array}{l}n=6 \\
n=3 \\
n=6\end{array}$ & $\begin{array}{l}\text { Azul } \\
\text { Transparente e bege } \\
\text { Transparente e preto }\end{array}$ & $\begin{array}{l}40 \% \\
20 \% \\
40 \%\end{array}$ \\
\hline \multicolumn{4}{|l|}{ Amostra 4} \\
\hline $\begin{array}{l}\text { Fio/fibra } \\
\text { Filme plástico } \\
\text { Fragmento plástico } \\
\text { Microesfera }\end{array}$ & $\begin{array}{l}n=3 \\
n=2 \\
n=1 \\
n=1\end{array}$ & $\begin{array}{l}\text { Azul e preto } \\
\text { Transparente e branco } \\
\text { Transparente } \\
\text { Branco }\end{array}$ & $\begin{array}{l}42,86 \% \\
28,56 \% \\
14,29 \% \\
14,29 \%\end{array}$ \\
\hline \multicolumn{4}{|l|}{ Amostra 5} \\
\hline $\begin{array}{l}\text { Fio/fibra } \\
\text { Filme plástico } \\
\text { Fragmento plástico }\end{array}$ & $\begin{array}{l}n=4 \\
n=2 \\
n=2\end{array}$ & $\begin{array}{l}\text { Preto e transparente } \\
\text { Transparente } \\
\text { Transparente }\end{array}$ & $\begin{array}{l}50 \% \\
25 \% \\
25 \%\end{array}$ \\
\hline
\end{tabular}

Fonte: Elaborado pelos autores. 
Tabela 4: Descrição de MP em mexilhões análise 2.

\begin{tabular}{|c|c|c|c|}
\hline & Quantidade & Cor predominante & $\begin{array}{l}\text { Frequência } \\
\text { relativa }\end{array}$ \\
\hline \multicolumn{4}{|l|}{ Amostra 1} \\
\hline Fio/fibra & $\mathrm{n}=1$ & Transparente & $100 \%$ \\
\hline \multicolumn{4}{|l|}{ Amostra 2} \\
\hline $\begin{array}{l}\text { Fio/fibra } \\
\text { Filme plástico } \\
\text { Fragmento plástico } \\
\text { Microesfera }\end{array}$ & $\begin{array}{l}n=5 \\
n=6 \\
n=2 \\
n=3\end{array}$ & $\begin{array}{l}\text { Preto } \\
\text { Transparente bege } \\
\text { Preto e prateado } \\
\text { Transparente }\end{array}$ & $\begin{array}{l}31,25 \% \\
37,50 \% \\
12,50 \% \\
18,75 \%\end{array}$ \\
\hline \multicolumn{4}{|l|}{ Amostra 3} \\
\hline $\begin{array}{l}\text { Fio/fibra } \\
\text { Filme plástico } \\
\text { Fragmento plástico }\end{array}$ & $\begin{array}{l}n=1 \\
n=1 \\
n=2\end{array}$ & $\begin{array}{l}\text { Preto } \\
\text { Transparente bege } \\
\text { Transparente }\end{array}$ & $\begin{array}{l}25 \% \\
25 \% \\
50 \%\end{array}$ \\
\hline \multicolumn{4}{|l|}{ Amostra 4} \\
\hline $\begin{array}{l}\text { Fio/fibra } \\
\text { Filme plástico } \\
\text { Fragmento plástico }\end{array}$ & $\begin{array}{l}n=3 \\
n=4 \\
n=3\end{array}$ & $\begin{array}{l}\text { Preto } \\
\text { Branco e transparente } \\
\text { Preto e preto e prateado }\end{array}$ & $\begin{array}{l}30 \% \\
40 \% \\
30 \%\end{array}$ \\
\hline \multicolumn{4}{|l|}{ Amostra 5} \\
\hline $\begin{array}{l}\text { Fio/fibra } \\
\text { Filme plástico }\end{array}$ & $\begin{array}{l}n=2 \\
n=2\end{array}$ & $\begin{array}{l}\text { Preto e roxo } \\
\text { Transparente }\end{array}$ & $\begin{array}{l}50 \% \\
50 \%\end{array}$ \\
\hline
\end{tabular}

Fonte: Elaborado pelos autores.

As fibras são um dos tipos de MP mais consumidos por várias espécies marinhas como anelídeos, bivalves, peixes, anêmonas entre outros (GUSMÃO et al., 2016; MORAIS et al., 2020; SILVA-CAVALCANTI et al., 2017), e podem estar associados a maior proximidade de centros urbanos (SILVA-CAVALCANTI et al., 2017), e processo de lavagem de roupas (BELZAGUI et al., 2019). As fibras na cor azul também foram as mais observadas em diferentes peixes e em diferentes níveis tróficos em estuários, associados a detritos plásticos originados da pesca (DANTAS et al., 2012; FERREIRA et al., 2016).

As Figuras 4 e 5 apresentam a percentagem de cada um dos tipos de MP encontrado de acordo com o seu formato, tendo como maior incidência no experimento 1 o fio/fibra e no experimento 2 o filme. 
Figura 4: Gráfico da quantidade de MP encontrada de acordo com o seu formato na Análise 1.

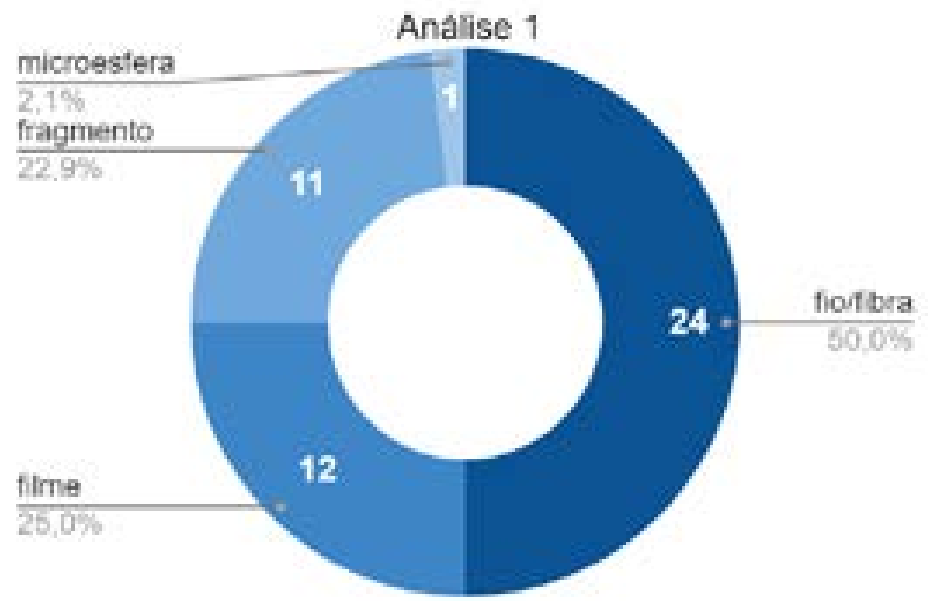

Fonte: Elaborado pelos autores.

Figura 5: Gráfico da quantidade de MP encontrada de acordo com o seu formato na Análise 2.

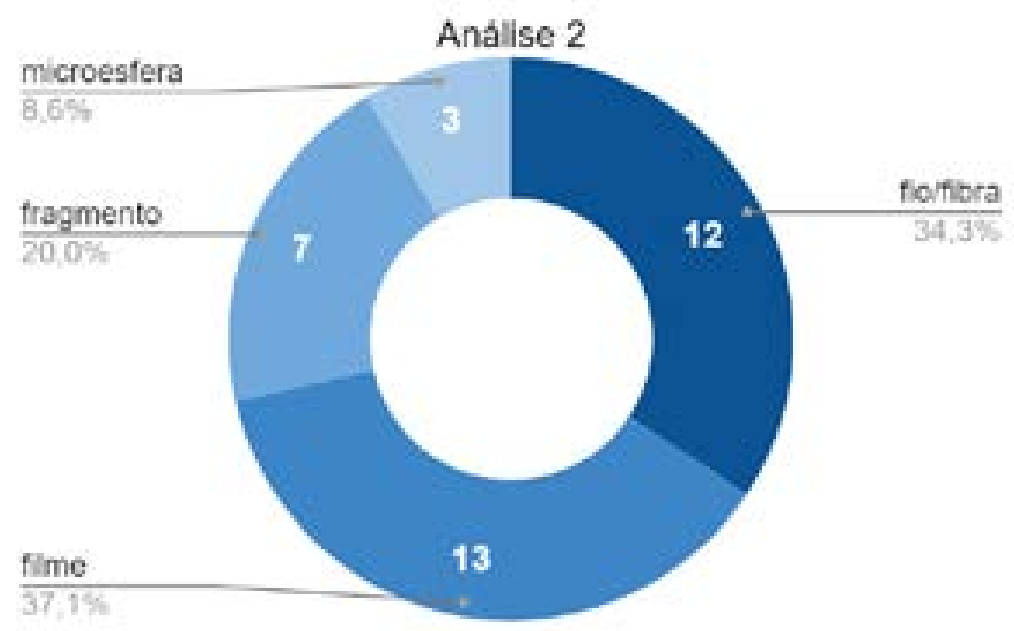

Fonte: Elaborado pelos autores.

Em ambas as análises foram encontradas partículas de cores distintas, sendo comum, em todas elas, fios/fibras transparentes e pretos; filmes transparentes, beges e brancos; fragmentos pretos e transparentes. Em relação às quantidades, foi observada uma grande variação de uma análise para a outra, onde na Análise 1 foram observados, 
respectivamente, mais fios/fibras, filmes e fragmentos, enquanto na Análise 2 os filmes tiveram a maior incidência, seguido dos fios/fibras e fragmentos. Nota-se que as partículas microplásticas foram encontradas em todas as amostras.

Li et al. (2015), em estudo semelhante realizado com bivalves de 8 espécies diferentes, observou os fios/fibras como sendo o MP mais recorrente, contabilizando um número superior a metade do total, e a menor incidência ficou por conta das microesferas, que foram encontradas em pequenas quantidades em seis do total de oito espécies estudadas, tendo, assim, resultados que coincidem aos encontrados no presente estudo.

4.3 Quatidade de MP por mexilhão e por peso úmido

Na Tabela 5 são apresentadas as quantidades encontradas de partículas de MP por mexilhão e por peso úmido nas análises 1 e 2.

Tabela 5: Quantidade de MP por mexilhão e por peso úmido

\begin{tabular}{|c|c|c|c|c|}
\hline & \multicolumn{2}{|l|}{$1^{\mathrm{a}}$ análise } & \multicolumn{2}{|l|}{$2^{\mathrm{a}}$ análise } \\
\hline & $\begin{array}{l}\text { Por mexilhão } \\
\text { (itens ind-1) }\end{array}$ & $\begin{array}{l}\text { Por peso úmido } \\
\text { (itens } \mathrm{g}-1 \text { ) }\end{array}$ & $\begin{array}{l}\text { Por mexilhão } \\
\text { (ítens ind-1) }\end{array}$ & $\begin{array}{l}\text { Por peso úmido } \\
\text { (itens } \mathrm{g}-1 \text { ) }\end{array}$ \\
\hline Amostra 1 & 2,66 & * & 0,33 & 0,08 \\
\hline Amostra 2 & 3,33 & 0,40 & 5,33 & 1,16 \\
\hline Amostra 3 & 5,00 & 0,58 & 3,33 & 0,24 \\
\hline Amostra 4 & 2,33 & 0,56 & 2,33 & 0,59 \\
\hline Amostra 5 & 2,66 & 0,51 & 1,33 & 0,25 \\
\hline
\end{tabular}

Fonte: Elaborado pelos autores.

*Dado perdido.

Podemos observar a presença dos polímeros por mexilhão em todas as amostras de ambas as análises, com valores que variaram de 2,33 itens ind-1 a 5,00 itens ind-1 e 0,33 itens ind-1 e 5,33 itens ind-1, nas análises 1 e 2, respectivamente. Quando observados os valores por peso úmido, observa-se na análise 2 , grande disparidade entre as amostras, com valores variando entre 0,08 itens g-1 a 1,16 itens g-1 de MP, bem como menor variação na análise 1, com valores entre 0,40 itens g-1 e 0,58 itens g-1 de MP. As concentrações de MP encontradas nos mexilhões estão estritamente relacionadas ao comportamento, fisiologia 
e hábitos alimentares dos bivalves, bem como ao ambiente em que vivem (LI et al., 2015) e isto pode ser comprovado a partir da crescente poluição por MP reportada no Brasil a partir dos anos 2000 (CASTRO; SILVA; ARAÚJO, 2018).

Os resultados do presente trabalho indicam concentrações de microplásticos por grama até 4 vezes maiores daquelas encontradas na Europa (VAN CAUWENBERGHE et al., 2014). Já ao longo do litoral da China, Qu et al. (2018) observaram que bivalves M. edulis e $P$. vidiris apresentavam entre 1,52 a 5,36 itens $\mathrm{g}-1$ (peso úmido), e esses valores estavam correlacionados às águas circundantes. Nesse sentido, diferenças metodológicas de análise e exposição aos MP podem influenciar nas concentrações observadas. A produção mundial de plástico é distribuída de forma irregular sendo 51\% produzido no Ásia, com a China responsável por 30\%, 18\% produzido na Nafta, 17\% na Europa, 4\% na América do Sul, e 10\% entre as demais localidades (PLASTIC EUROPE, 2019). Apesar disso, os valores encontrados de MP em bivalves podem estar relacionados não apenas a produção e consumo de plástico em diferentes localidades, mas também a forma de descarte, a qualidade de manejo de resíduos, e ao transporte transfronteiriço (KRELLING et al., 2019; LIMA et al., 2020) que faz com que regiões possam ter diferentes quantidades de MP. Dessa forma, é possível que exista, efetivamente, grandes concentrações de MPS em amostras de mexilhões Perna perna e no litoral brasileiro. No Brasil, apenas Santana et al. (2016) verificou a presença de MP em 75\% das amostras avaliadas ao longo da Baía de Santos em mexilhões Perna perna, e que a contaminação não ocorre de forma sistemática e prevista no estuário.

Os mexilhões expostos em costões rochosos da encosta das praias estão susceptíveis a MP que se degradam tanto através de intemperismos como frio, calor, raios UV, mas também com a ação mecânica das ondas, que além de fragmentar os plásticos, associados aos eventos de maré e ventos, transportam os MP para as praias que servem tanto de sumidouro como fonte para esses microdetritos (RODRÍGUEZ et al., 2020). Esses movimentos de maré, ondas e ventos, biodisponibilizam MP a biota ao longo dos costões próximos às praias. Espécies de anêmonas Bunodosoma cangicum também tem ingerido MP em substrato rochoso no norte do Brasil, acredita-se que esse consumo esteja associado a contaminação direta e indireta, uma vez que as espécies se encontram em área de entre-maré, e necessitam de ampla absorção de água durante a maré baixa, além de possuírem hábitos alimentares generalistas e oportunistas (MORAIS et al., 2020). Caranguejos Pachygrapsus transversus (GIBBES, 1850) presentes em recifes também tem consumido microplásticos de forma direta e indireta através do consumo de bivalves, e que $47,4 \%$ da amostra havia consumido MP, 
principalmente fibras de nylon azul. Os resultados do estudo demonstraram que a ingestão de MP tem influenciado o Fator de condição de Le Cren (Kn) e por consequência o estado de saúde geral da espécie (DE BARROS et al., 2020).

\section{Considerações finais}

O presente trabalho foi desenvolvido com a utilização de mexilhões Perna perna coletados e comercializados no município de Matinhos (PR), sendo possível concluir que há a ingestão de microplásticos por esses bivalves selvagens.

Os resultados desse trabalho são os primeiros indicativos de um problema ambiental e para a saúde humana que pode ter maior severidade (i.e. outros poluentes associados aos microplásticos) e apresentar maior abrangência geográfica, ultrapassando os limites municipais (i.e. litoral do Paraná). Considerando isso, é importante que estudos mais abrangentes sejam realizados. Em relação a severidade, como apenas o método de inspeção visual de MP foi utilizado neste trabalho, sugere-se que futuros estudos adotem análise complementares para identificação mais precisa dos polímeros e poluentes associados, utilizando análises químicas por técnicas de espectrometria (FTIR e Raman) e outras técnicas analíticas.

Em relação a sua abrangência geográfica, sugere-se que estudos de escala estadual ou regional sejam realizados para determinar o status da contaminação dos bivalves no litoral paranaense. Este estudo contribui com indícios de poluição por MP no litoral do estado, contudo, estudos ainda mais robustos podem não apenas contribuir com a compreensão da seriedade e da extensão da poluição por microplásticos no litoral paranaense, mas também permitir a comparação com outras regiões do Brasil e do mundo.

\section{Referências}

ALVES, TP; SCHRAMM, MA, PROENÇA, LAO, PINTO, TO, MAFRA LL Jr. Interannual variability in Dinophysis spp. abundance and toxin accumulation in farmed mussels (Perna perna) in a subtropical estuary. Environ Monit Assess. 2018 May 5;190(6):329. doi: 10.1007/s10661-018-6699-y. PMID: 29730718.

ANDRADY, Anthony L.. Microplastics in the marine environment. Marine Pollution Bulletin, [s.l.], v. 62, n. 8, p.1596-1605, ago. 2011.

ANDRADY, Anthony L.. The plastic in microplastics: A review. Marine Pollution Bulletin, [s.l.], v. 119, n. 1, p.1222, jun. 2017. 
ARAÚJO, A. P. DA C., \& MALAFAIA, G. Can short exposure to polyethylene microplastics change tadpoles' behavior? A study conducted with neotropical tadpole species belonging to order anura (Physalaemus cuvieri). Journal of Hazardous Materials, 391(January), 122214, 2020.

ASCER, Liv Goldstein. Efeitos de Microplásticos na Fisiologia do Mexilhão Perna perna (Bivalvia:Mytilidae). 2015. 88 f. Dissertação (Mestrado) - Curso de Ciências, Fisiologia Geral, Universidade de São Paulo, São Paulo, 2015.

BARROS,M. S. F., CALADO, T.C.S. C., SILVA, A. S.S, ANTOS, E.V. Ingestion of plastic debris affects feeding intensity in the rocky shore crab Pachygrapsus transversus Gibbes 1850 (Brachyura: Grapsidae). International Journal of Biodiversity and Conservation, 12(1), 113-117, 2020.

BELZAGUI, F., CRESPI, M., ÁLVAREZ, A., GUTIÉRREZ-BOUZÁN, C., \& VILASECA, M. Microplastics' emissions: Microfibers' detachment from textile garments. Environmental Pollution, 248, 1028-1035, 2019.

BOTTERELL, Z. L. R., BEAUMONT, N., DORRINGTON, T., STEINKE, M., THOMPSON, R. C., \& LINDEQUE, P. K. Bioavailability and effects of microplastics on marine zooplankton: A review. Environmental Pollution, Plymouth, v. 245, p.98-110, out. 2019.

BOUCHER, J; FRIOT, D. Primary Microplastics in the Oceans: a Global Evaluation of Sources. Gland: IUCN, 2017. 46 p. Disponível em: <http://www.iucn.org/resources/publications>. Acesso em: 10 out. 2019.

BRASIL. Ministério da Pesca e Turismo. Manual do MPA para o Programa Nacional de Controle Higiênico Sanitário de Moluscos Bivalves - PNCMB. 2a ed. Brasília: CGSAP/DEMOC/SEMOC/MAPA, 2014.

BRÅTE, I. L. N., HURLEY, R., IVERSEN, K., BEYER, J., THOMAS, K. V., STEINDAL, C. C., GREEN, N. W., OLSEN, M., \& LUSHER, A. (2018). Mytilus spp. as sentinels for monitoring microplastic pollution in Norwegian coastal waters: A qualitative and quantitative study. Environmental Pollution, 243, 383-393, 2018.

BROWNE, M.A., UNDERWOOD, A.J., CHAPMAN, M.G., WILLIAMS, R. THOMPSCON, R.C., FRANEKER, A.A.V. Linking effects of anthropogenic debris to ecological impacts. Proceedings Of The Royal Society B: Biological Sciences, [s.I.], v. 282, n. 1807, p. 2014-2929, 22 maio 2015.

CARON, A. G. M., THOMAS, C. R., BERRY, K. L. E., MOTTI, C. A., ARIEL, E., \& BRODIE, J. E. Validation of an optimised protocol for quantification of microplastics in heterogenous samples: A case study using green turtle chyme. Methodsx, [s.l.], v. 5, p.812-823, 2018.

CASTRO, R. O., SILVA, M. L., ARAÚJO, F. V., Review on microplastic studies in Brazilian aquatic ecosystems. Ocean \& Coastal Management, [s.I.], v. 165, p.385-400, nov. 2018.

COSTA, M., SUL, J.A.I., LIMA, A.R.A., BARLETTA, M. Contaminação do mar brasileiro por resíduos plásticos: impactos e perspectivas. 5o. Congresso Brasileiro de Biologia Marinha. Porto de Galinhas, 17-21 Maio de 2015.

DANTAS, D. V., BARLETTA, M., \& DA COSTA, M. F. The seasonal and spatial patterns of ingestion of polyfilament nylon fragments by estuarine drums (Sciaenidae). Environmental Science and Pollution Research, 19(2), 600-606, 2012.

DE BARROS, M. S. F., DOS SANTOS CALADO, T. C., \& DE SÁ L. C. A. M Plastic ingestion lead to reduced body condition and modified diet patterns in the rocky shore crab Pachygrapsus transversus (Gibbes, 1850) (Brachyura: Grapsidae). Marine Pollution Bulletin, 156(April), 111249, 2020.

DERRAIK, Jose G B. The pollution of the marine environment by plastic debris. Marine Pollution Bulletin, vol. 44, no. 9, p. 842-852, 2002. https://doi.org/10.1016/s0025-326x(02)00220-5. 
EUROPE PLASTIC. Association of plastic manufeacturers. DE. Carbon neutral print productions. 2019. Disponível em :< https://www.plasticseurope.org/application/files/9715/7129/9584/FINAL_web_version_Plastics_the_ facts2019_14102019.pdf>. Acessado em 30 de setembro de 2019.

EVANGELISTA-BARRETO, N.S.; SOUSA, O.V; VIEIRA, R.H.S.F. Moluscos bivalves: organismos bioindicadores da qualidade microbiológica das águas. Revista Brasileira de Higiene e Sanidade Animal, [s.I.], v. 2, n. 2, p.17$29,2008$.

FERREIRA, G. V. B., BARLETTA, M., LIMA, A. R. A., DANTAS, D. V., JUSTINO, A. K. S., \& COSTA, M. F. Plastic debris contamination in the life cycle of Acoupa weakfish (Cynoscion acoupa) in a tropical estuary. ICES Journal of Marine Science: Journal Du Conseil, 73(10), 2695-2707, 2016.

FRIAS, João Pedro Garcez Luís de. Microplásticos: o "presente" envenenado. 2010. 150 f. Dissertação (Mestrado) - Curso de Engenharia do Ambiente, Universidade Nova de Lisboa, Lisboa, 2010.

GALGANI, F.; OOSTERBAAN, L; POITOU, I; HANKE, G; THOMPSON, R; AMATO, E; JANSSEN, C; GALGANI, F; FLEET, D; FRANEKER, J Van; KATSANEVAKIS, S; MAES, T. Marine Strategy Framework Directive: Task Group 10 Report Marine Litter. [S. I.: s. n.], 2010. https://doi.org/10.2788/86941.

GALGANI, F; LEAUTE, J P; MOGUEDET, P; SOUPLET, A; VERIN, Y; CARPENTIER, A; GORAGUER, H; LATROUITE, D; ANDRAL, B; CADIOU, Y. Litter on the sea floor along European coasts. Marine pollution bulletin, vol. 40, no. 6, p. $516-527,2000$.

GESAMP. Sources, fate and effects of microplastics in the marine environment: part two of a global assessment. London: International Maritime Organization, 2016. 220 p.

GOOGLE MAPS (2021). Matinhos - PR. Disponível em https://www.google.com/maps/place/Matinhos++PR/@-25.7762455,-48.6411226,11z/data=!3m1!4b1!4m5!3m4!1 s0x94dbedf599045eb7:0xe4ee4b3401ddb77 $5 ! 8 \mathrm{~m} 2 ! 3 \mathrm{~d}-25.8167999 ! 4 d-48.5383013$. Consultado em 05/07/2021.

GORMAN, D., MOREIRA, F. T., TURRA, A., FONTENELLE, F. R., COMBI, T., BÍCEGO, M. C., \& DE CASTRO MARTINS, C. Organic contamination of beached plastic pellets in the South Atlantic: Risk assessments can benefit by considering spatial gradients. Chemosphere, 223, 608-615, 2019

GUEBERT-BARTHOLO, F. M.; BARLETTA, M.; COSTA, M. F.; MONTEIRO-FILHO, E. L. A. Using gut contents to assess foraging patterns of juvenile green turtles Chelonia mydas in the Paranaguá Estuary, Brazil. Endangered Species Research, v. 13, n. 2, p. 131-143, 2011.

GUSMÃO, F., DOMENICO, M. DI, AMARAL, A. C. Z., MARTÍNEZ, A., GONZALEZ, B. C., WORSAAE, K., IVAR DO SUL, J. A., \& CUNHA LANA, P. DA. In situ ingestion of microfibres by meiofauna from sandy beaches. Environmental Pollution, 216, 584-590, 2016.

HARTMANN, N.B., HÜFFER, T., THOMPSON, R.C., HASSELLÖV, M., VERSCHOOR, A., DAUGAARD, A.E., RIST, S., KARLSSON, T.; BRENNHOLT, N.,, COLE, M., HERRLING, M.P., HESS, M.C., IVLEVA, N.P., LUSHER, A.L., WAGNER, M. Are We Speaking the Same Language? Recommendations for a Definition and Categorization Framework for Plastic Debris. Environmental Science \& Technology, [s.I.], v. 53, n. 3, p.1039-1047, 4 jan. 2019.

INFOESCOLA (2021). Mapa do Brasil com Estados. Disponível em https://www.infoescola.com/wp-content/ uploads/2019/07/mapa-do-brasil-estados-branco-comlegenda.jpg, Acesso em 05 de julho de 2021.

JAMBECK, Jenna R., GEYER, Roland, WILCOX, Chris, SIEGLER, Theodore R., PERRYMAN, Miriam, ANDRADY, Anthony, NARAYAN, Ramani, KARA, Lei A. Plastic waste inputs from land into the ocean. Science, [s.I.], v. 347, n. 6223, p.768-771, 12 fev. 2015. American Association for the Advancement of Science (AAAS).

KARBALAEI, S., GOLIESKARDI, A., HAMZAH, H. B., ABDULWAHID, S., HANACHI, P., WALKER, T. R., \& KARAMI, A. Abundance and characteristics of microplastics in commercial marine fish from Malaysia. Marine Pollution Bulletin, [s.I.], v. 148, p.5-15, nov. 2019. 
KRELLING, AP;SOUZA, M. M.;WILLIAMS, A.T.;TURRA, A. Transboundary movement of marine litter in an estuarine gradient: Evaluating sources and sinks using hydrodynamic modelling and ground truthing estimates. Marine Pollution Bulletin, v. 119, n. 1, p. 48-63, 2017a.

KRELLING, ALLAN PAUL; WILLIAMS, A. T.; TURRA, A. Differences in perception and reaction of tourist groups to beach marine debris that can influence a loss of tourism revenue in coastal areas. Marine Policy, v. 85, $n$. November, p. 87-99, 2017b.

KRELLING, A. P.; TURRA, A. Influence of oceanographic and meteorological events on the quantity and quality of marine debris along an estuarine gradient. Marine Pollution Bulletin, p. 282-298, 2019.

KOELMANS A.A, NOR, N.H.M., HERMSEN, E., KOOI, M. , MINTENIG, S. M., DE FRANCE J. Microplastics in freshwaters and drinking water: Critical review and assessment of data quality. Water Research, [s.I.], v. 155, p.410-422, maio 2019.

KOLM, H. E., \& ABSHER, T. M. Bacterial density and coliform organisms in waters and oysters of Paranagua Estuarine Complex, Parana, Brazil AN - 19795684; 8805774. Boletim Do Instituto de Pesca Sao Paulo, 34(1), 49-59, 2008.

LI, J., LUSHER, A. L., ROTCHELL, J. M., DEUDERO, S., TURRA, A., LISE, I., BRÅTE, N., SUN, C., HOSSAIN, M. S., LI, Q., KOLANDHASAMY, P., \& SHI, H. Using mussel as a global bioindicator of coastal microplastic. Environmental Pollution, 244, 522-533, 2019.

LI, J., YANG, D., LI, L., JABEEN, K., \& SHI, H. Microplastics in commercial bivalves from China. Environmental Pollution, [s.I.], v. 207, p.190-195, dez. 2015.

LIMA, A. R. A.; SILVA, M. D.; POSSATTO, F. E.; FERREIRA, G. V. B.; KRELLING, A. P. Plastic Contamination in Brazilian Freshwater and Coastal Environments: A Source-to-Sea Transboundary Approach. The Handbook of Environmental Chemistry, 2020.

MARTINEZ, D. I., \& de OLIVEIRA, A. J. F. C. Faecal bacteria in Perna perna (Linnaeus, 1758) (Mollusca: Bivalvia) for biomonitoring coastal waters and seafood quality. Brazilian Journal of Oceanography, 58 (SPEC. ISSUE 3), 29-35., 2010

MEYER, U., HAGEN, W., \& MEDEIROS, C. Mercury in a northeastern Brazilian mangrove area, a case study: Potential of the mangrove oyster Crassostrea rhizophorae as bioindicator for mercury. Marine Biology, 131(1), 113-121, 1998.

MORAIS, L. M. S., SARTI, F., CHELAZZI, D., CINCINELLI, A., GIARRIZZO, T., \& MARTINELLI Filho, J. E. The sea anemone Bunodosoma cangicum as a potential biomonitor for microplastics contamination on the Brazilian Amazon coast. Environmental Pollution, 265, 114817. 2020.

NEVES, Diogo Fernando Pereira. Lixo marinho nos fundos oceânicos e a sua ingestão por peixes da costa portuguesa. 2013. 77 f. Dissertação (Mestrado) - Curso de Engenharia do Ambiente Perfil de Gestão e Sistemas Ambientais, Universidade Nova de Lisboa, Lisboa, 2013.

PHUONG, N.N. ZALOUK-VERGNOUUX, A., KAMARI, A., MOUNEYRAC, C., AMIARD, F., POIRIER, L., \& LAGARDE, F. Quantification and characterization of microplastics in blue mussels (Mytilus edulis): protocol setup and preliminary data on the contamination of the French Atlantic coast. Environmental Science And Pollution Research, [s.l.], v. 25, n. 7, p.6135-6144,. 2018.

POSSATTO, F. E.; SPACH, H. L.; CATTANI, A. P.; et al. Marine debris in a World Heritage Listed Brazilian estuary. Marine Pollution Bulletin, v. 91, n. 2, p. 548-553, 2015. 
PRATA, J. C., DA COSTA, J. P., LOPES, I., DUARTE, A. C., \& ROCHA-SANTOS, T. Effects of microplastics on microalgae populations: A critical review. Science of the Total Environment, Portugal, v. 665, p.400-405, fev. 2019.

QU, X., SU, L., LI, H., LIANG, M., \& SHI, H. Assessing the relationship between the abundance and properties of microplastics in water and in mussels. Science of the Total Environment, 621, 679-686, 2018.

RODRÍGUEZ, C., FOSSATTI, M., CARRIZO, D., SÁNCHEZ-GARCÍA, L., TEIXEIRA DE MELLO, F., WEINSTEIN, F., \& LOZOYA, J. P. Mesoplastics and large microplastics along a use gradient on the Uruguay Atlantic coast: Types, sources, fates, and chemical loads. Science of the Total Environment, 721, 2020.

SANTANA, M. F.M., ASCER, L. G., CUSTÓDIO, M. R., MORIRA, F. T., \& TURRA, A. (2016). Microplastic contamination in natural mussel beds from a Brazilian urbanized coastal region: Rapid evaluation through bioassessment. Marine Pollution Bulletin, 106(1-2), 183-189, 2016.

SANTANA, M. F. M., MOREIRA, F. T., \& TURRA, A.Trophic transference of microplastics under a low exposure scenario: Insights on the likelihood of particle cascading along marine food-webs. Marine Pollution Bulletin, 121(1-2), 154-159, 2017.

SCOTT, N., PORTER, A., SANTILLO, D., SIMPSON, H., LLOYD-WILLIAMS, S., \& LEWIS, C. Particle characteristics of microplastics contaminating the mussel Mytilus edulis and their surrounding environments. Marine Pollution Bulletin, [s.l.], v. 146, p.125-133, set. 2019.

SILVA-CAVALCANTI, J. S., SILVA, J. D. B., FRANÇA, E. J. DE, ARAÚJO, M. C. B. DE, \& GUSMÃO, F. Microplastics ingestion by a common tropical freshwater fishing resource. Environmental Pollution, 221, 218-226, 2017.

SMITH, M., LOVE, D.C., ROCHMAN, C.M., NEFF, R.A. Microplastics in Seafood and the Implications for Human Health. Current Environmental Health Reports, [s.I.], v. 5, n. 3, p.375-386, 16 ago. 2018.

SOUZA, R. V. de; PETCOV, H. F. D.. Comércio legal de moluscos bivalves. Florianópolis, SC; Epagri, 58 p. , 2013 (Epagri, Boletim Didático, no 95).

SUPLICY, F. M. Plano Estratégico para Desenvolvimento Sustentável da Maricultura Catarinense (2018- 2028). Florianópolis: Epagri, 2018. 89 p.

TIBIRIÇÁ, C.E.; LEITE, I.; BATISTA, T. V.V; FERNANDES, L.F.; CHOMÉRAT, N.; HERVE, F.; HESS., MAFRA, L.L. Ostreopsis cf. ovata Bloom in Currais, Brazil: phylogeny, toxin profile and contamination of mussels and marine plastic litter. Toxins, 11, 446, 2019.

THOMPSON, R., MOORE, C., ANDRADY, A., GRAGORY, M., TAKADA, H. \& WEISBERG, S. New Directions in Plastic Debris. Science, [s.l.], v. 310, n. 5751, p.1117-1119, 18 nov. 2005. American Association for the Advancement of Science (AAAS).

UNEP, Valuing Plastics: The Business Case for Measuring, Managing and Disclosing Plastic Use in the Consumer Goods Industry. p. 116, 2014.

UNEP. Marine litter. Disponível em: <https://www.unenvironment.org/explore-topics/oceans-seas/what-wedo/working-regional-seas/marine-litter>. Acesso em: 10 out. 2019.

VAN CAUWENBERGHE, L., \& JANSSEN, C. R. Microplastics in bivalves cultured for human consumption. Environmental Pollution, 193, 65-70, 2014.

VIEIRA, K. S., BAPTISTA NETO, J. A., CRAPEZ, M. A. C., GAYLARDE, C., PIERRI, B. da S., SALDAÑA-SERRANO, M., BAINY, A. C. D., NOGUEIRA, D. J., \& FONSECA, E. M. Occurrence of microplastics and heavy metals accumulation in native oysters Crassostrea Gasar in the Paranaguá estuarine system, Brazil. Marine Pollution Bulletin, 166 (February), 2021. 
WANG, W., GAO, H., JIN, S., LI, R., NA, G.. The ecotoxicological effects of microplastics on aquatic food web, from primary producer to human: A review. Ecotoxicology And Environmental Safety, [s.I.], v. 173, p.110-117, maio 2019.

WIDMER, W. M., \& REIS, R. A. An experimental evaluation of the effectiveness of beach ashtrays in preventing marine contamination. Brazilian Archives of Biology and Technology, 53(5), 1205-1216, 2010.

ZANTIS, L. J., CARROLL, E. L., NELMS, S. E., \& BOSKER, T. Marine mammals and microplastics: A systematic review and call for standardisation. Environmental Pollution, 269, 11614, 2021.

ZHU, C., LI, D., SUN, Y., ZHENG, X., PENG, X., ZHENG, K., HU, B., LUO, X., \& MAI, B. Plastic debris in marine birds from an island located in the South China Sea. Marine Pollution Bulletin, [s.I.], v. 149, dez. 2019. 